\title{
DoctorBio: Um Estudo de Caso sobre a Utilização de Recursos de Realidade Aumentada no Ensino de Ciências Biológicas
}

\author{
${ }^{1}$ Faculdade Anhanguera - Pelotas, RS - Brazil \\ ${ }^{2}$ Research Group - Wearable Technology (WeTech) \\ Instituto Federal Sul Rio-grandense (IFSul) - Pelotas, RS - Brazil \\ ${ }^{3}$ Centro de Desenvolvimento Tecnológico (CDTec) \\ Universidade Federal de Pelotas (UFPel) - Pelotas, RS - Brazil \\ leandrodoctorbio@gmail.com, marcelo@ifsul.edu.br, \\ $\{a p v \cdot v a s c o n c e l l o s$, tatiana\}@inf.ufpel.edu.br
}

Leandro Araujo ${ }^{1}$, Marcelo B. Machado ${ }^{2}$, Ana Priscila Vasconcellos ${ }^{3}$, Tatiana Aires Tavares ${ }^{3}$

\begin{abstract}
Digital technologies are part of the modern world generating a larger number of users. In Education field, these technologies are also taking place into pedagogical processes. The current work presents an implementation of solutions based in digital technologies applied to teaching-learning process through an application for smart phones. So, we introduce DoctorBio project, a way to to make different the use of smartphones inside classrooms as an useful device for teaching Biological Sciences. DoctorBio uses Aurasma. Aurasma is a platform for augmented reality which turns possible making textbooks come to life. Using augmented reality, DoctorBio make mobile devices an object of learning helping students and teachers to interact with biology content in an attractive and enjoy full way.
\end{abstract}

Resumo. As tecnologias digitais fazem parte do mundo moderno gerando um maior número de usuários. No campo da Educação, essas tecnologias também estão ocorrendo em processos pedagógicos. O presente trabalho apresenta uma implementação de soluções baseadas em tecnologias digitais aplicadas ao processo de ensino-aprendizagem por meio de uma aplicação para smartphones. Assim, apresentamos DoctorBio projeto, uma maneira de fazer diferente o uso de smartphones dentro de salas de aula como um dispositivo útil para o ensino de Ciências Biológicas. DoctorBio usa Aurasma, uma plataforma para realidade aumentada que torna possível fazer livros didáticos ganharem vida. Usando a realidade aumentada, o sistema DoctorBio faz com que os dispositivos móveis sejam um objeto de aprendizagem ajudando estudantes e professores a interagir com o conteúdo de biologia de uma forma atraente e divertida.

\section{Introdução}

A forma lúdica de ensinar, não só nas séries iniciais, sempre foi uma das práticas mais prazerosas de abordar assuntos complexos para o processo de aprendizagem. Um exemplo ocorre na área de Ciências Biológicas, que tem como objetivo principal estudar os seres vivos e todas as suas mais variadas características de vida. Devido a uma grande 
quantidade de matéria, com nomes e estruturas de múltiplas dimensões e níveis de complexidade, percebe-se nos alunos certo grau de desinteresse, ou ainda, pouca curiosidade sobre o tema [Prieto et al. 2005].

O lúdico deixa-se levar por uma certa sensação de liberdade espontânea, sem que aconteça limites para desbravar o desconhecido, onde não existem rígidas formas de contextualizar e avaliar determinados assuntos impostos, deixando assim, a principal causa descontraída, podendo quebrar qualquer tipo de padrões diante de uma atividade. Para [Freinet 1998], a ludicidade era: “(...) um estado de bem-estar que é a exacerbação de nossa necessidade de viver, de subir e de perdurar ao longo do tempo. Atinge a zona superior do nosso ser e só pode ser comparada à impressão que temos por uns instantes de participar de uma ordem superior cuja potência sobre-humana nos ilumina”.

$\mathrm{Na}$ medida em que atravessamos gerações, os meios lúdicos de ensino e aprendizagem tendem a se modernizar conforme a realidade na qual estão inseridos, e dessa forma, ampliam-se as possibilidades de serem abertos novos caminhos para a ludicidade. Com o crescimento dos meios tecnológicos, surgem cada dia mais ferramentas e objetos de aprendizagem capazes de auxiliar a educação, e ainda que, podem ser usadas para muitas atividades lúdicas [Piaget 2007]. Pesquisas indicam que o aluno pode ter diferentes tipos de relação com o estudo do conteúdo. Muitos dos estudantes estão preocupados com a repercussão externa do seu trabalho; as notas que vão tirar, a necessidade de passar nos exames, interesse em atender as demandas e agradar o professor. Memorizam fatos, informações, geralmente de formas desconexas apenas para atender as mínimas exigências escolares ou para um sentido prático profissional numa visão atómistica do problema [Krasilchik 2004].

Nesse sentido, as tecnologias podem ser um meio de promover formas mais lúdicas de ensino, tornando o processo mais dinâmico e atraente aos alunos, uma vez que vivemos em um mundo cada vez mais moderno e voltado para os meios tecnológicos, e na medida em que esses meios são inseridos na sociedade geram impactos diretos na educação. É nesse ponto que concentra-se a problematização deste trabalho, no sentido de trazer o celular como aliado para as atividades em sala de aula. Em outras palavras, conectar os meios tecnológicos com a realidade no âmbito educacional, traçando assim um paralelo de que a qualidade do ensino pode tomar rumos mais atuais e muito mais atrativos [Dallabonna and Mendes 2004], [Castro et al. 2014]. A tecnologia cada vez mais ganha espaço no cenário educacional, pois agora ela passa a atuar diretamente como instrumento de aprendizagem, e assim, conquista espaço também em um âmbito social. $\mathrm{O}$ celular ou qualquer dispositivo móvel de uso geral, por exemplo, é um recurso cada vez mais tecnológico e com mais popularidade, tanto na sociedade quanto na sala de aula. Desfrutar da internet através de dispositivos móveis é cada vez mais comum, atingindo a todas as idades, desde muito cedo. O uso permanente de celulares dentro da sala de aula, por vezes, acaba sendo o grande inimigo dos professores. Um exemplo disso é a lei n $12.7301^{1}$, de 11/10/2007 do Estado de São Paulo, que proíbe o uso do telefone celular nos estabelecimentos de ensino do Estado, durante o horário de aula.

É nesse ponto que se concentra a problematização desse trabalho, no sentido de reconhecer a presença do smartphone no meio acadêmico e o trazer para dentro da sala

\footnotetext{
${ }^{1}$ http://www.al.sp.gov.br/norma/?id=74333
} 
VI Congresso Brasileiro de Informática na Educação (CBIE 2017)

Anais do XXIII Workshop de Informática na Escola (WIE 2017)

de aula. Em outras palavras, conectar os meios tecnológicos com a realidade no âmbito educacional, traçando assim um paralelo de que a qualidade do ensino pode tomar rumos mais atuais e muito mais atrativos.

Este artigo tem por objetivo apresentar resultados da investigação do uso de recursos em realidade aumentada através de celulares como objeto de aprendizagem de forma a transformá-lo em um auxiliar para professores e alunos. Para tanto, é apresentada uma estratégia na tentativa de tornar o celular como um objeto de desenvolvimento de tecnologia para o ensino de Biologia, intitulado como DoctorBio. O DoctorBio seria um canal de interatividade desenvolvido em realidade aumentada como artefato complementar ao livro texto de sala de aula. Para o desenvolvimento desse canal de interatividade sugerimos a utilização da plataforma Aurasma [Aurasma 2016] , uma plataforma para realidade aumentada compatível com dispositivos móveis.

\section{Materiais e Métodos}

A tecnologia enraiza-se cada vez mais no cotidiano das pessoas, tanto profissional como pessoal. Na educação, observa-se o mesmo fenômeno. Novas tecnologias são desenvolvidas para facilitar o processo de ensino-aprendizagem [Domingos 2012]. Contemplando os meios lúdicos de ensino, a tecnologia também é uma grande aliada nessa técnica tão explorada por grandes estudiosos, permite que se possa criar infinitas formas de recorrer os caminhos da compreensão, através de jogos, brincadeiras e diferentes maneiras de visualizar os conteúdos. Torna com certeza, a interação entre crianças e jovens muito mais atrativa, e facilita o acesso ao mundo do conhecimento por intermédio dessas ferramentas.

Sites, blogs, fotologs, games, softwares de comunicação instantânea e telefones celulares com câmeras digitais (foto e vídeo) e computadores (menores e mais potentes) são instrumentos cada vez mais utilizados por jovens de todas as idades [Luna 2009]. Tradicionalmente, os professores tem seu método prático de ministrar sua aula, por uma infinidade de motivos que os levam a não mudar sua prática mais tradicional, porém, muitos já recorrem a outras formas mais ousadas e atrativas com o emprego da tecnologia.

As novas tecnologias de informação e comunicação são ferramentas indispensáveis no contexto da educação. Para aderir às novas tecnologias é necessário definir critérios e competências técnicas e um grande conhecimento específico para aliar os conteúdos a serem trabalhados com as inovações tecnológicas do mundo moderno [Vasconcelos et al. 2003, Gama and Porto 2014, Ferreira 1993].

No presente trabalho utilizamos as tecnologias voltadas à Realidade Aumentada (RA). Realidade Aumentada se trata de uma nova tecnologia onde os usuários podem interagir com objetos reais e virtuais através da superposição de camadas virtuais. A RA pode ser definida como uma realidade mista, para isto, ela necessita de três componentes básicos, sendo eles, a combinação da realidade real e virtual, possuir uma interatividade em tempo real e ser registrada em três dimensões [Azuma 2001]. É nesse ponto que a realidade aumentada é uma tecnologia interessante para a educação, podendo prestar subsídios para inúmeras atividades criativas de construir uma tarefa direcionada ao ensino-aprendizagem. A realidade aumentada é uma tendência no mercado corporativo e tem sido, cada vez mais, objeto de estudo.

$\mathrm{Na}$ Educação, a RA apresenta potencial para proporcionar desenvolvimento de uma nova visão na forma de instigar o aluno a ser o agente de seu próprio 
VI Congresso Brasileiro de Informática na Educação (CBIE 2017)

Anais do XXIII Workshop de Informática na Escola (WIE 2017)

aprendizado [Domingos 2012]. Um exemplo desse fato é a experiência discutida em [Barreto et al. 2015] que demonstra o grande potencial da utilização da RA em contextos de acessibilidade. Para os autores a versatilidade no acesso e na exibição do conteúdo das camadas virtuais são pontos chave para uma interação mais transparente. Por sua vez, a interação mais transparente que quase surpreende o usuário, é um passo em direção à acessibilidade. A ferramenta utilizada para implementar os cartões interativos [Barreto et al. 2015] foi o Aurasma [Aurasma 2016].

No intuito de implementar os recursos de realidade aumentada, utilizamos o Aurasma, uma plataforma digital que oferece um aplicativo para dispositivos móveis compatível com os sistemas operacionais Android e IOS. A escolha do Aurasma para o desenvolvimento deste trabalho levou em conta fatores tais como a praticidade apresentada pela plataforma, ao permitir a incorporação de recursos de autoria e exibição em um mesmo ambiente. O Aurasma [Aurasma 2016] é uma plataforma que suporta autoria, publicação e visualização de camadas virtuais que utiliza tecnologia de reconhecimento de imagem e projeta objetos virtuais através da câmera do celular ou do tablet. Para o desenvolvimento o Aurasma disponibiliza um SDK (kit de desenvolvimento) que facilita a autoria das camadas virtuais (chamadas de "auras"). O funcionamento das camadas virtuais está atrelado ao mapeamento de uma imagem-gatilho (do ambiente real) e qualquer outra imagem, vídeo ou objeto tridimensional. Desta maneira, o aplicativo mistura o real como o virtual e gera um conteúdo interativo. O Aurasma é um aplicativo de realidade aumentada compatível com ambientes Android e IOS. Seu principal requisito é ter acesso com a internet, pois ele trabalha com marcações visuais em objetos do mundo real que por sua vez são ligadas em camadas, ou "Auras". As Auras podem apresentar objetos multimídias como imagens, vídeos ou objetos 3D, conforme [Sales et al. ].

O método utilizado foi baseado na metodologia de desenvolvimento de objetos de aprendizagem com o foco na aprendizagem significativa proposta por [Tavares et al. 2007]. Optou-se por uma equipe de desenvolvimento multidisciplinar incorporando profissionais das áreas de Design, Computação e Ciências Biológicas, conforme ilustrado na Figura 1.

As principais etapas de realização do projeto:

- Etapa 1: Definição do tema. Todos os envolvidos (Designer, programador e professor biólogo) identificam a temática a ser abordada (conteúdo da aula).

- Etapa 2: Identificação do público-alvo. O professor (Biólogo) define o público alvo, isto é, a turma.

- Etapa 3: Elaboração do conteúdo. Todos trabalham na elaboração do conteúdo digital que será adotado. Primeiramente o professor define o estilo de conteúdo desejado (um jogo ou uma animação, por exemplo) e então é criado um esboço do conteúdo da aula.

- Etapa 4: Elaboração dos objetos digitais: De posse do esboço da etapa anterior, os objetos digitais são criados e/ou editados.

- Etapa 5: Desenvolvimento da camada virtual. É criado uma camada virtual para o canal DoctorBio incluindo os objetos digitais criados na etapa anterior.

- Etapa 6: Integração, ajustes e testes. Para finalização da camada criada todos os envolvidos testam a camada virtual. Adicionalmente foram construídos dois protótipos: um protótipo para interatividade com o livro-texto já utilizado com 
VI Congresso Brasileiro de Informática na Educação (CBIE 2017)

Anais do XXIII Workshop de Informática na Escola (WIE 2017)

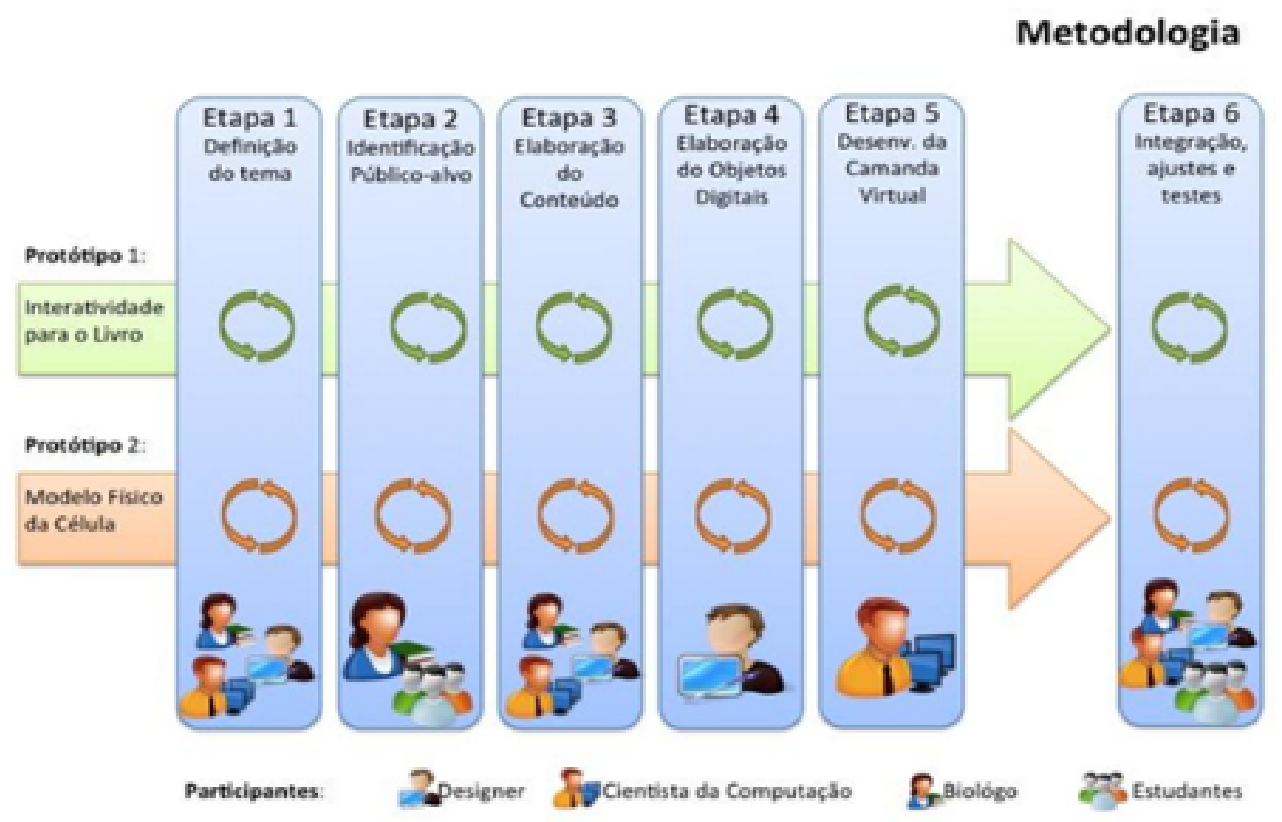

Figura 1. Metodologia em desenvolvimento de objetos.

os alunos em sala de aula e outra utilizando um modelo físico feito em e.v.a. representando uma célula e suas organelas

\section{Resultados e Discussão}

O canal DoctorBio agrupa um total de 6 camadas virtuais abordando os conteúdos: célula vegetal, célula animal e vírus, respectivamente. A Figura 2 mostra o modelo da célula em EVA (Figura 2 -a) e a edição da camada, mais precisamente, a identificação da trigger (do modelo físico) pela ferramenta de autoria disponibilizada pelo Aurasma. Todas as camadas desenvolvidas utilizam como base uma trigger que consiste em uma imagem do mundo real que será utilizada como gatilho para disparar o conteúdo virtual correspondente.

Na Figura 3 pode-se observar o detalhamento de uma camada virtual sendo acessada pelo aluno através de um tablet para interação. Neste exemplo é utilizado ao invés do modelo em EVA (acetato-vinilo de etileno), Figura 2, o próprio livro texto do aluno. O livro texto utilizado foi Ciências Vida na Terra de Fernando Gewandsnajder [Gewandsznajder 2002].

O canal DoctorBio foi aplicado como instrumento de aprendizagem na disciplina de Ciências com alunos da $7^{\text {a }}$ série do ensino fundamental, da Escola Estadual Cassiano do Nascimento, localizada na cidade de Pelotas/RS. A turma escolhida possuía 19 alunos, 9 meninos e 10 meninas. Como instrumento de avaliação foram aplicados dois questionários (pré e pós-teste) de perguntas abertas e de livre respostas.

Os alunos foram abordados em dois momentos: primeiramente responderam a um questionário antes de conhecer o objeto de aprendizagem (fase de pré-teste). E em 


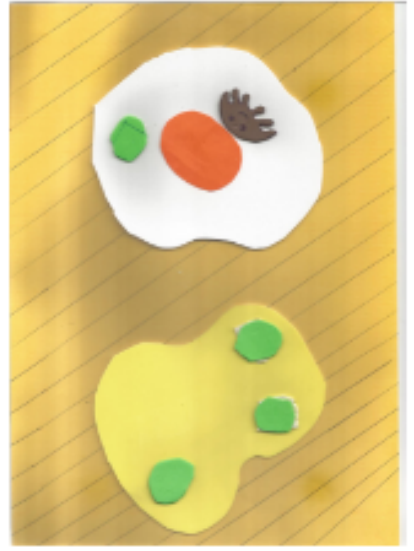

(a)

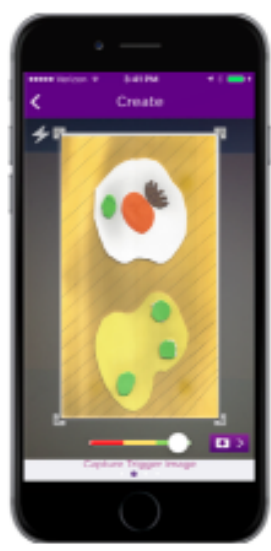

(b)

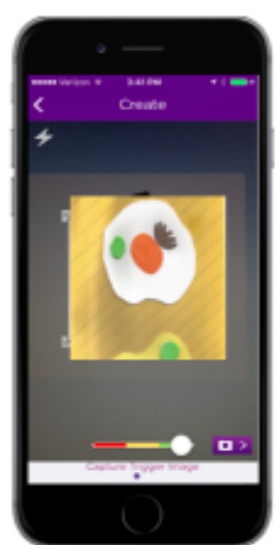

(c)

Figura 2. Camadas Virtuais com EVA. Em (a) estruturas em EVA, em (b) e (c) edição da camada virtual utilizando o AURASMA. Fonte: Arquivo pessoal

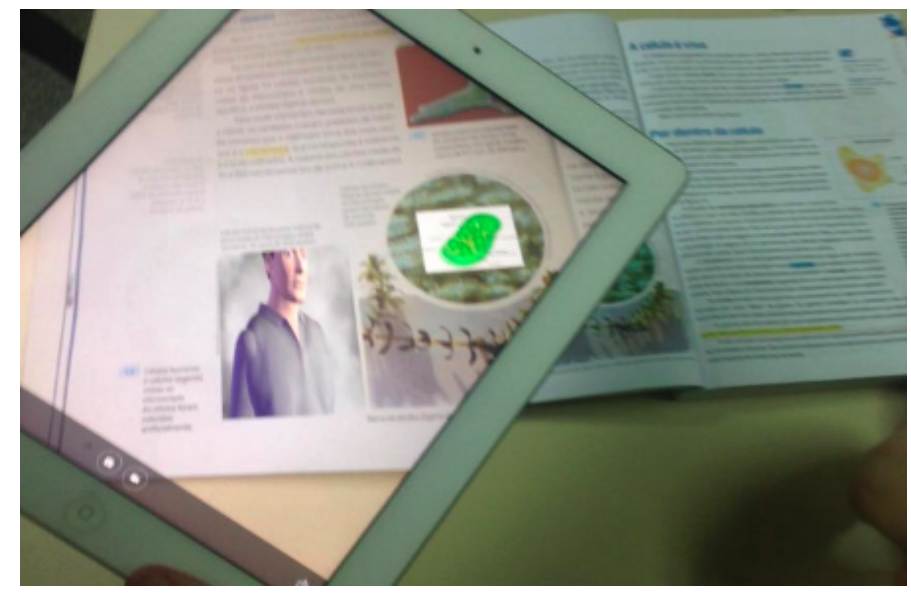

Figura 3. Utilização da camada virtual em desenvolvimento com o livro texto. Fonte: Arquivo pessoal

um segundo momento, após conhecerem e utilizarem o canal DoctorBio, responderam à segunda rodada de perguntas (fase pós-teste).

A Figura 5 mostra os resultados obtidos na aplicação do questionário pré-teste. A faixa etária dos alunos variou de 12 a 18 anos (vide 6). Destaca-se que a maioria dos alunos, 82,4 por cento, possui celular e que 76,5 por cento também costuma levar o smartphone para a sala de aula, Figura 5 (b) e (c). Em um índice de desempenho, 60 por cento dos alunos classificaram a experiência com o DoctorBio como boa e 40 por cento identificaram a experiência como ótima.

\section{Conclusão}

Tomando como base os estudos feitos nesta pesquisa, é possível sugerir novas experiências reais em sala de aula, onde aluno e professor buscam novas interações com os meios tecnológicos. O uso dos smartphones, que é uma tecnologia de alta popularidade, sugere uma aula extremamente motivadora e interativa, onde a ferramenta que tanto causa desconforto para o professor em sala de aula acaba se tornando um grande aliado. 
VI Congresso Brasileiro de Informática na Educação (CBIE 2017)

Anais do XXIII Workshop de Informática na Escola (WIE 2017)

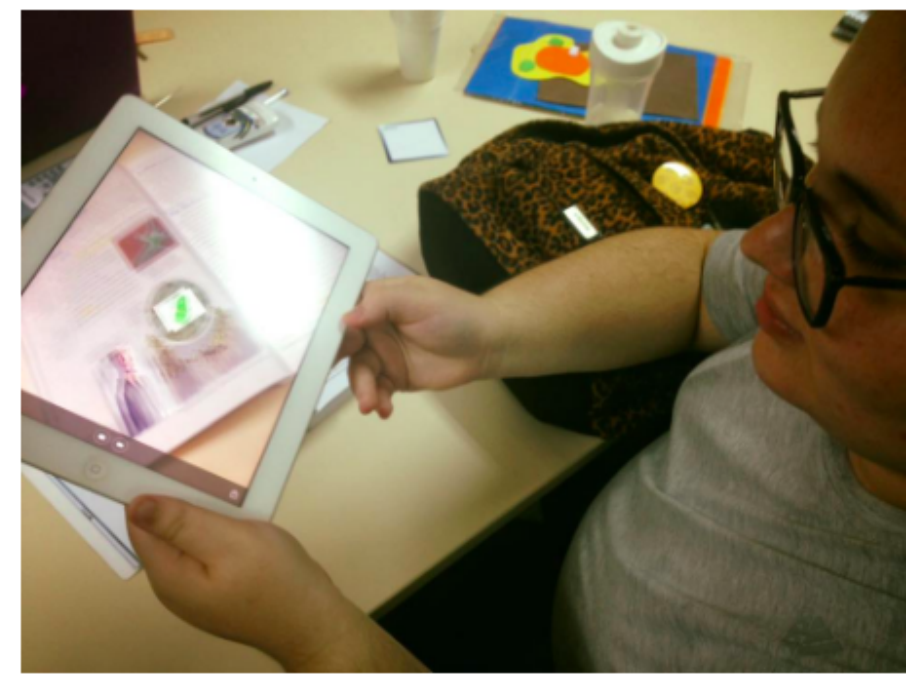

Figura 4. Utilização da camada virtual desenvolvida com o livro texto. Fonte: Arquivo pessoal

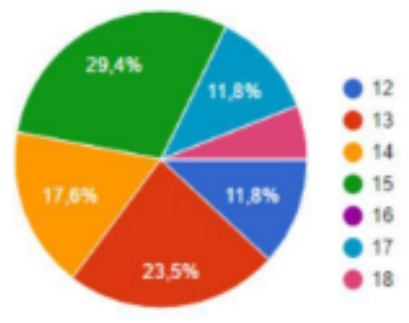

(a)

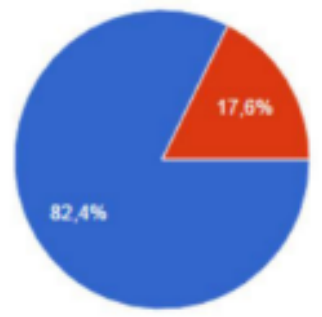

(b)

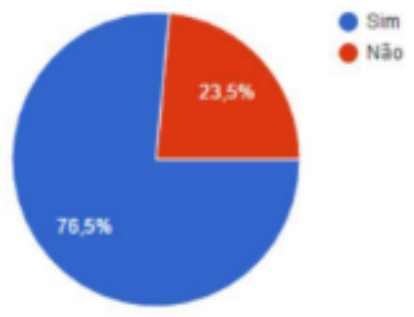

(c)

Figura 5. Resultados do Questionário Pré-Teste. Fonte: Arquivo pessoal

O uso de realidade aumentada nos livros textos através dos dispositivos móveis em geral, ajuda a tornar o ensino de Ciências Biológicas mais palpável aos olhos dos alunos, uma vez que, facilita a compreensão e resgata o seu interesse em aprender de uma forma divertida e prazerosa. As possibilidades desse tipo de solução para enriquecer o ensino de Ciências Biológicas são inúmeras e podem tornar o smartphone uma espécie de laboratório para sala de aula.

Os resultados obtidos em sala de aula mostraram-se satisfatórios. O uso da realidade aumentada através dos dispositivos móveis possibilitou uma experiência motivadora, tanto para os alunos, quanto para os professores. Os alunos ficaram muito animados com a possibilidade de utilizarem um dispositivo para tornar as aulas mais interativas, animadas e colaborativas.

Destacamos algumas impressões registradas pelos alunos quando solicitados para registrar sua opinião:

"Eu achei legal, mais interativo e todos cooperaram na aula"(Aluno 14, idade: 13 anos). 


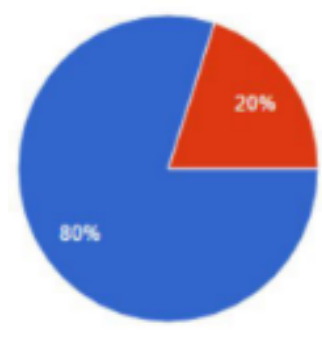

(a)
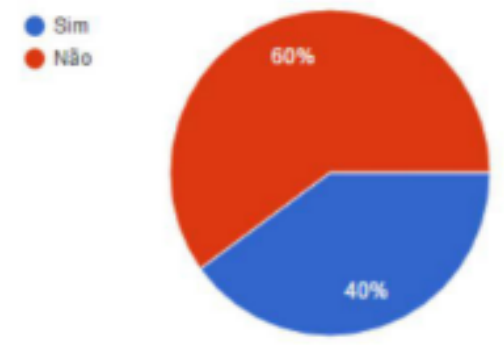

(b)

Figura 6. Resultados do Questionário Pós-Teste. Fonte: Arquivo pessoal

"Eu achei muito bom porque nem todo professor ensina desta maneira."(Aluno 10, idade 13 anos).

Percebe-se que, ao aliar a tecnologia com a educação, pode-se ganhar um grande reforço pertinente às práticas pedagógicas. Ao fazer a atribuição dos dispositivos móveis, tal como o smartphone, como ferramentas de aprendizagem, ganha-se não só a atenção e o interesse dos alunos, mas também a ampliação da qualidade de educação ofertada à sociedade como um todo.

Os índices de aprovação deste primeiro experimento remetem-se a continuação dos estudos sobre esta nova metodologia associada a tecnologia.

Para a sequência dos trabalhos, destacam-se dois pontos cruciais que balizarão a continuidade decorrente desta primeira experiência. A questão de que a grande maioria dos alunos têm e carregam consigo para aula o aparelho celular ou smartphone, e a construção de novos "cases" em realidade aumentada. Um mundo de possibilidades é colocado em marcha como, avatares que falam sobre o tema, janelas de gravações feitas pelos professores, mais animações, abertura de links com mais informações, interatividade com outras pessoas conectadas, etc.

Enfim, o objetivo não é só apresentar e realçar o conteúdo mas abrir uma ponte para aprofundamentos e desdobramentos deste conteúdo com outras situações que, antecipadamente podem fugir da percepção. Aqui pode-se realmente incentivar a criatividade e a liberdade na formação dos alunos, pois neste contexto ele poderá criar caminhos e ou propô-los da forma como lhe convier.

Também caberia fazer uso de um grupo de controle para que se possa, numa experimentação mais longa, avaliar o resultado efetivo com relação a assimilação do conteúdo pelos alunos e as novas ressignificações com relação à disciplina em foco oriundas da aplicação desta tecnologia.

\section{Referências}

Aurasma (2016). Hewlett-packard development company. Disponível em: https://www.aurasma.com/.

Azuma, R. (2001). Recent advances in augmented reality. In v .21, n., editor, IEEE Computer Graphics and Applications, pages 34-47. Disponível em: 
VI Congresso Brasileiro de Informática na Educação (CBIE 2017)

Anais do XXIII Workshop de Informática na Escola (WIE 2017)

http://www.cs.unc.edu/ azuma/cga2001.pdf.

Barreto, H., Barreto, L., Carvalho, J., Dias, M., Lopes, R., , and Tavares, T. (2015). Muito além do papel: Uma experiência de design e avaliação de realidade aumentada para cartões impressos. In Anais do Workshop de Realidade Virtual e Aumentada (WRVA). Disponível em: http://www.lbd.dcc.ufmg.br/colecoes/wrva/2015/025.pdf.

Castro, R., Medeiros, A., Santos, F., Tavares, T., and Fonseca, I. (2014). A utilização de interação natural em ambientes tridimensionais para treinamento no setor elétrico. In Anais dos Workshops do Congresso Brasileiro de Informática na Educação.

Dallabonna, S. and Mendes, S. (2004). O lúdico na educação infantil: jogar, brincar, uma forma de educar. In Revista de divulgação técnico-científica do ICPG 1.4, pages $107-112$.

Domingos, D. (2012). A realidade aumentada como objetos de aprendizagem: As novas tecnologias na ead. In XII Colóquio Internacional de Gestão Universitária, pages $1-11$.

Ferreira, B. (1993). Minidicionário da língua portuguesa. Edição Revista e Ampliada, 3 edition.

Freinet, C. (1998). A educação do trabalho. São Paulo-SP : Martins Fontes, 1 edition.

Gama, E. and Porto, C. (2014). Realidade aumentada à serviço da educação: Uma experiência com o aurasma. In Sempesq. Disponível em https://eventos.set.edu.br/index.php/sempesq/article/view/940.

Gewandsznajder, F. (2002). Ciências: A vida na Terra. São Paulo: Áticas.

Krasilchik, M. (2004). Prática de ensino de biologia. EduUSP, 1 edition.

Luna, M. (2009). Tecnologia e ludicidade. Disponível em: http://brinqueeaprenda.blogspot.com.br/2009/05/tecnologia-e-ludicidade-pormarlucio.html.

Piaget, J. (2007). Epistemologia genética. Tradução de Álvaro Cabral. São Paulo-SP : Martins Fontes, 3 edition.

Prieto, L., Trevisan, M., Danesi, M., and Falkembach, G. (2005). Uso das tecnologias digitais em atividades didáticas nas séries iniciais. In Revista Novas Tecnologias na Educação. CINTED UFRGS.

Sales, A., Siebra, B., Djohnnatha, C., Ferreira, W., and Tavares, T. A. Cineguide: uma aplicação multimídia com realidade aumentada para informativo de cinema.

Tavares, R., Anjos, L., and Gouveia, T. (2007). Metodologia de desenvolvimento de objetos de aprendizagem com foco na aprendizagem significativa. In XVII Simpósio Brasileiro de Informática na Educação, page 8.

Vasconcelos, C., Praia, J., and Almeida, L. (2003). Teorias de aprendizagem e o ensino/aprendizagem das ciências: da instrução à aprendizagem. In Psicologia Escolar e Educacional. 